\title{
Del trastorn específic del llenguatge (TEL) al trastorn del desenvolupament del llenguatge (TDL): un canvi de concepció sobre els trastorns del llenguatge
}

\author{
Eva Aguilar-Mediavilla, Lucía Buil-Legaz, Àngels Esteller-Cano i \\ Josep A. Pérez-Castelló
}

Investigació en Desenvolupament, Educació i Llenguatge (I+DEL); Universitat de les Illes Balears

eva.aguilar@uib.es

Recepció: 26/03/2018, acceptació: 11/06/2019

Resum: El trastorn específic del llenguatge (TEL) es va definir com una alteració greu en l'adquisició i el desenvolupament del llenguatge, que podia afectar tant la comprensió com l'expressió, en absència de determinades causes i condicions. No obstant això, en els darrers anys els estudis han constatat que les dificultats del llenguatge rarament són aillades, sinó que van acompanyades d'altres dificultats cognitives relacionades amb les habilitats de processament del llenguatge. A més, els requisits diagnòstics tant restrictius del TEL han fet que, en l'àmbit clínic, molts infants no es puguin diagnosticar d'aquest trastorn tot i les seves mancances, i que, per tant, no puguin rebre l'atenció que necessiten. Això ha fet que recentment es promogués un estudi de consens en l'àmbit anglosaxó (el projecte CATALISE) que ha comportat un canvi en l'etiqueta diagnòstica, de TEL a trastorn del desenvolupament del llenguatge (TDL), però també, i més important, un canvi de perspectiva diagnòstica, que ha passat d'estar enfocada en el diagnòstic estàtic a una de més dinàmica, basada en criteris de persistència temporal i en les necessitats d'intervenció. Aquest fet ha comportat, de manera secundària, un canvi en la classificació de les dificultats del llenguatge, que ha passat de basar-se en característiques diagnòstiques a basar-se en el tipus d'intervenció que requereix cadascuna d'aquestes dificultats. En aquest article abordarem, des d'aquesta nova concepció, la definició i els criteris diagnòstics d'aquest trastorn, les seves característiques i els seus signes precoços d'alerta.

Paraules clau: trastorn específic del llenguatge, trastorn del desenvolupament del llenguatge, signes d'alerta.

Del trastorno específico del lenguaje (TEL) al trastorno de desarrollo del lenguaje (TDL): un cambio de concepción sobre los trastornos del lenguaje

Resumen: El trastorno específico del lenguaje (TEL) se definió como una alteración grave en la adquisición y el desarrollo del lenguaje, que podía afectar tanto a la comprensión como la expresión, en ausencia de determinadas causas y condiciones. No obstante, en los últimos años los estudios han constatado que las dificultades del lenguaje raramente son aisladas, sino que van acompañadas de otras dificultades cognitivas relacionadas con las habilidades de procesamiento del lenguaje. Además, su definición, con unos requisitos diagnósticos muy restrictivos, ha hecho que, en el ámbito clínico, muchos niños no puedan ser diagnosticados de este trastorno a pesar de sus carencias, y que por tanto no puedan 
recibir la atención que necesitan. Esto ha hecho que recientemente se promoviera un estudio de consenso (el proyecto CATALISE) que ha supuesto un cambio en la etiqueta diagnóstica, de TEL a trastorno del desarrollo del lenguaje (TDL), pero también, y más importante, un cambio de perspectiva diagnóstica, que ha pasado de estar enfocada en el diagnóstico estático a una más dinámica, basada en criterios de persistencia temporal y en las necesidades de intervención. Este hecho ha comportado, de forma secundaria, un cambio en la clasificación de las dificultades del lenguaje, que ha pasado de basarse en características diagnósticas a basarse en el tipo de intervención que requiere cada una de estas dificultades. En este artículo abordaremos, desde esta nueva concepción, la definición y los criterios diagnósticos de este trastorno, sus características y sus signos precoces de alerta.

Palabras clave: trastorno específico del lenguaje, trastorno de desarrollo del lenguaje, signos de alerta.

\section{From specific language impairment (SLI) to developmental language disorder (DLD): a change in language disorder conception}

Abstract: Specific language impairment (SLI) was defined as a serious alteration in the acquisition and development of language, which could affect both comprehension and expression, in the absence of certain causes and conditions. However, in recent years, studies have found that language difficulties are rarely isolated, but that they are accompanied by other cognitive difficulties related to the language processing abilities. In addition, its definition, with very restrictive diagnostic requirements, has led that, in the clinical field, many children cannot be diagnosed with this disorder despite their deficits, and therefore cannot receive the care they need. This has recently led the promotion of a consensus study (the CATALISE project), which has led to a change in the diagnostic tag, from SLI to developmental language disorder (DLD), but also, and more importantly, to a change in the diagnostic perspective, which has gone from being focused on the static diagnosis to a more dynamic one, based on criteria of temporary persistence and intervention needs. This fact has led, in a secondary way, to a change in the classification of language difficulties, which has gone from being based on diagnostic characteristics to be based on the type of intervention that each of these difficulties requires. In this article, we will address, from this new conception, the definition and the diagnostic criteria of this disorder, its characteristics and its early warning signs.

Keywords: language specific impairment, developmental language disorder, alert signs.

\section{UN CANVI EN LA CONCEPCIÓ SOBRE ELS TRASTORNS \\ DEL LLENGUATGE}

La classificació de les dificultats de la veu, la parla, el llenguatge i la comunicació ha estat un tema complex des dels seus inicis. Aquesta dificultat ve donada per la falta de consens entre les diferents disciplines (psicologia, lingüística, medicina o logopèdia), fet que comporta enfocaments teòrics, concepcions, visions i ús de termes diferents (Bishop, Snowling, Thompson, Greenhalgh i CATALISE consortium 2016; Bishop 2017). No hem d'oblidar que una classificació comporta un enfocament teòric, que a la vegada té implicacions en la intervenció pràctica i pot influir en les polítiques dels serveis vinculats a les famílies i persones amb patologies del neurodesenvolupament.

En aquest sentit, quan parlem de les dificultats de la veu, la parla, el llenguatge i la comunicació en els trastorns del neurodesenvolupament, hem de tenir presents dos aspectes importants. D'una banda, que hem de veure l'infant com un ésser complet que es 
desenvolupa globalment, és a dir, que no és estàtic ni dividit en diferents components de desenvolupament. De l'altra, que existeixen diferents plànols d'explicació o d'enfocament d'aquestes dificultats: conductual, cognitiu, biològic i contextual (Morton 2005). Aquests plànols són complementaris entre si, no contraposats, i permeten veure les dificultats des de diferents punts de vista. A partir de les evidències podem observar que aquests plànols afecten les persones que presenten patologies del neurodesenvolupament de diferents maneres i amb diferents intensitats durant el seu desenvolupament; així, per exemple, cada dificultat del neurodesenvolupament es veurà afectada en diferent mesura pels aspectes geneticobiològics, els cognitius i els ambientals, el que produiran canvis en cascada en la conducta d'aquestes persones durant el seu desenvolupament.

Per aquesta raó, el neuroconstructivisme (Karmiloff-Smith 1998) explica la influència del propi desenvolupament en les patologies del neurodesenvolupament, entenent l'individu com un ésser global i canviant a causa de la interacció entre l'individu (biològic) i el context en què es desenvolupa. Aquesta aportació teòrica considera que el cervell adult està format per diversos components amb una funció cognitiva específica (per exemple, el llenguatge), i que aquesta modularització sorgeix durant el propi desenvolupament a través d'una especialització funcional progressiva de les diferents funcions del cervell. Aquesta especialització es genera a partir de les especificacions genètiques, la interacció amb l'ambient i la connexió entre les diferents funcions que van sorgint (D'Souza i Filippi 2017). Per tant, aquesta teoria posa en evidència la importància de les interaccions entre els mòduls del cervell i emfatitza una visió dinàmica del desenvolupament en persones amb dificultats o sense. En el cas de les dificultats del neurodesenvolupament, a causa de la interacció entre aquests mòduls, les zones més afectades poden impactar, mitjançant un efecte en cascada, en altres parts que no són objecte primari d'afectació. És a dir, els dèficits en nivells de desenvolupament bàsic poden desencadenar efectes posteriors en nombrosos aspectes del desenvolupament més complexes.

Partint d'aquesta teoria és més fàcil entendre els canvis de concepció que ha patit el trastorn específic del llenguatge o TEL (ara anomenat internacionalment trastorn del desenvolupament del llenguatge, TDL, o Developmental Language Disorder, DLD) i, de retruc, la classificació de les dificultats de la veu, la parla, el llenguatge i la comunicació. El TEL es va definir, als anys 8o, com una alteració greu en l'adquisició i el desenvolupament del llenguatge, que podia afectar tant la comprensió com l'expressió lingüística, sense que hi hagués cap dels motius que generalment explicaven aquestes dificultats, com el retard mental, la discapacitat auditiva, el dany cerebral i/o problemes socioemocionals i comportamentals (Stark i Tallal 1981). No obstant això, en els darrers anys els estudis han constatat que els infants amb TEL rarament mostren dificultats aillades en el llenguatge, sinó que també s'observen altres dificultats cognitives relacionades amb les habilitats de processament del llenguatge (Bishop 1992, Johnston 1999, Marton 2008, Montgomery 2003), així com dificultats sensoriomotrius (Hill 1998; Hill, Bishop i Nimmo-Smith 1998; Schwartz, Mody i Petinou 1998; Zelaznik i Goffman 2010), acadèmiques (Catts, Fey, Tomblin, i Zhang 2002; Fazio 1996; Goulandris, Snowling i Walker 2000) i socioemocionals (Hegde 1996; Leonard 1998; St Clair, Pickles, Durkin i Conti-Ramsden 2011). Entre les dificultats que s'han trobat es poden destacar les següents:

- Dificultats en habilitats cognitives, tant de processament fonològic, consciència fonològica, memòria de treball fonològic i denominació ràpida, com d'atenció auditiva, activitats simbòliques, imaginativitat mental, raonament complex i càlcul matemàtic, atenció i funcions executives (Bishop 1992; Fazio 1996; Henry, Messer i Nash 2012; Johnston 1999; Marton 2008; Montgomery 2003; Schwartz, Mody i Petinou 1998).

- Dificultats lectores (Catts et al. 2002, Goulandris et al. 2000). 
- Dificultats en funcions sensoriomotrius (per exemple, problemes de coordinació: Hill 1998, Hill et al. 1998).

- Dificultats emocionals, socials i conductuals (per exemple, St Clair et al. 2011).

De fet, l'evidència mostra que hi ha una alta comorbiditat amb altres trastorns del neurodesenvolupament, com ara la dislèxia (30-43\%; Alvarado i Pérez-Castelló 2018, Archibald, Oram, Cardy, Joanisse i Ansari 2013), la disortografia i disgrafia o la discalcúlia (34\%; Alvarado i Pérez-Castelló 2018, Archibald et al. 2013), el coeficient intel-lectual límit (32\%; Conti-Ramsden, Botting i Faragher 2001), els problemes de relació social (50\%; Conti-Ramsden, Botting i Faragher 2001), els problemes de conducta (54\%; Maggio, Grañana, Richaudeau, Torres, Giannotti i Suburo 2014), el dèficit de processament auditiu (16\%; Rocha-Muniz, Befi-Lopes i Schochat 2015), les dificultats sensoriomotrius (57\%; Hill 1998, Hill et al. 1998), els trastorns de la parla (70\%; Archibald i Gathercole 2006, Lindsay i Strand 2016), la prematuritat (19\%; Bosch-Galcerán, Agut Quijano i Busquets Ferrer 2018; Woods, Rieger, Wocadlo i Gordon 2014) i el trastorn per dèficit d'atenció amb hiperactivitat (TDAH, 30\%; Adrover-Roig, Sánchez-Azanza i López-Penadés 2018; Mueller i Tomblin 2012).

A més, la definició tant restrictiva de TEL ha fet que, en l'àmbit clínic, a molts infants no se'ls pugui classificar amb aquest diagnòstic, tot i les seves mancances, i que per tant no puguin rebre l'atenció que necessiten. Aquests fets varen fer que des del món angloparlant es dugués a terme un estudi de consens amb metodologia Delphi, liderat per la Dra. Dorothy V. M. Bishop, que va rebre el nom de Projecte CATALISE (Bishop et al. 2016; Bishop, Snowling, Thompson, Greenhalgh i CATALISE-2 consortium 2017). L'objectiu inicial del projecte era trobar un consens terminològic entre els professionals de diferents àmbits implicats en les dificultats del llenguatge (logopèdia, educació, psicologia clínica, psicologia educativa, associacions benèfiques, pediatria, psiquiatria, audiologia i escola) que facilités el diagnòstic de les dificultats del desenvolupament del llenguatge (Bishop et al. 2016, Bishop 2017). El projecte ha suposat finalment tres canvis importants. En primer lloc, ha suposat un canvi de nomenclatura i criteris classificatoris del TEL. Per tal d'evitar l'ús de la paraula específic, es passa de la nomenclatura tradicional de Specific Language Impairment (SLI; Trastorn Específic del Llenguatge o TEL) a adoptar el nou terme de Developmental Language Disorder (DLD; Trastorn del Desenvolupament del Llenguatge o TDL). En segon lloc, el projecte CATALISE ha suposat una nova visió de la classificació dels problemes de llenguatge que es basa en la necessitat que un infant ha de rebre tractament en la veu, la parla, el llenguatge i la comunicació, en comptes del compliment estricte d'uns criteris classificatoris. Finalment, ha comportat una nova classificació de les dificultats de la veu, la parla, el llenguatge i la comunicació basada en el tipus d'intervenció que necessita cada infant. És a dir, un trastorn es diferencia d'un altre sobretot pel tipus d'intervenció que comporta. Aquesta nova classificació distingeix entre les necessitats derivades del desconeixement de la llengua de l'entorn, els trastorns de la veu (disfonies), els trastorns de la fluència (disfèmies), els trastorns de la parla (disàrtria, dispràxia verbal, trastorns articulatoris, disglòssies i trastorns fonològics sense problemes de llenguatge) $\mathrm{i}$ els trastorns del llenguatge. Dins els trastorns del llenguatge diferencia entre els trastorns associats a alguna condició biomèdica coneguda (afàsia, epilèpsia adquirida amb afàsia, condicions genètiques, paràlisi cerebral, pèrdua auditiva, trastorn de l'espectre autista i discapacitat intel-lectual) i el TDL.

Per tant, el TDL (o DLD) és definit com una dificultat funcional del llenguatge que crea un obstacle a llarg termini en la vida diària per comunicar-se o per aprendre. El terme i la seva definició són menys restrictius que els del TEL. D’una banda, s'accepta que aquesta dificultat pot ser comòrbida amb problemes amb altres àrees com l'atenció (TDAH; Adrover-Roig et al. 2018, Mueller i Tomblin 2012), la motricitat (dispràxia i disàrtria; Hill 
1998, Hill et al. 1998), la lectoescriptura (dislèxia; Alvarado i Pérez-Castelló 2018, Archibald et al. 2013), la parla (trastorns articulatoris i fonològics; Archibald i Gathercole 2006), les funcions executives i la conducta adaptativa (Bishop 1992, Fazio 1996, Henry Messer i Nash 2012, Johnston 1999, Marton 2008, Montgomery 2003, Schwartz et al. 1998), els problemes conductuals (St Clair et al. 2011), el processament auditiu (dèficit de processament auditiu; Rocha-Muniz et al. 2015), i també amb nivells baixos d'intel-ligència no verbal (sense complir el diagnòstic de discapacitat intel-lectual; Conti-Ramsden et al. 2001), prematuritat (Bosch-Galcerán et al. 2018, Woods et al. 2014), i baix nivell sociocultural (Reilly, Wake, Ukoumunne, Bavin, Prior, Cini, Conway, Eadie i Bretherton 2010). D'altra banda, no posa un llindar en les deficiències en la competència lingüística a partir de les que es considera un TDL, sinó que es fa especial referència al fet que el problema lingüístic sigui a llarg termini i afecti la comunicació o els aprenentatges de l'infant.

\section{CRITERIS Diagnòstics I CARACTERÍSTIQUES DEL TRASTORN \\ DEL DESENVOLUPAMENT DEL LLENGUATGE EN INFANTS \\ BILINGÜES CATALÀ-CASTELLÀ}

Els criteris de diagnòstic de TDL inclouen un retard del llenguatge persistent, no resolt als 5 anys d'edat, que afecta la comunicació i/o aprenentatge de la vida quotidiana, sense una afecció mèdica que pugui explicar-la, com ara una lesió cerebral, condicions o trastorns genètics, una pèrdua auditiva, un trastorn de l'espectre autista o una discapacitat intel-lectual (Bishop et al. 2016). No obstant això, cal posar en relleu que el TDL pot aparèixer alhora que dèficits atencionals amb hiperactivitat o sense, dislèxia, trastorn del processament auditiu o un quocient intel-lectual baix que no justifica una discapacitat intel-lectual. Aquesta definició té una repercussió molt clara, i és que el diagnòstic s'ha d'entendre com un procés que s'inicia molt abans de l'edat de 5 anys i que continua fins i tot més enllà d'aquesta, amb revisions constants per constatar si aquestes dificultats lingüístiques estan afectant la comunicació o els aprenentatges de l'infant.

En concret, en el nostre entorn social bilingüe on els infants aprenen català i castellà alhora, els nens amb TDL mostren diverses dificultats des d'un punt de vista lingüístic. Les seves dificultats afecten especialment el component morfosintàctic, amb presència d'omissions en paraules de funció, errors de morfologia flexiva i una estructura sintàctica deficient (Serra-Raventós 2002, Serra-Raventós, Aguilar-Mediavilla i Sanz-Torrent 2002; Aguilar-Mediavilla, Sanz-Torrent i Serra-Raventós 2007). Però també s'observen dificultats en altres components lingüístics, com el fonològic, amb omissions de síl-labes àtones i reduccions de formes sil-làbiques (Aguilar-Mediavilla, Sanz-Torrent i SerraRaventós 2002; Aguilar-Mediavilla i Serra-Raventós 2006); lexicosemàntic, amb errors en l'ús de verbs i dificultats d'accés lèxic, i amb la pragmàtica, amb presència de poca coherència narrativa (Sanz-Torrent, Badia i Serra-Raventós 2008; Sanz-Torrent, Serrat Sellabona, Andreu Barrachina i Serra-Raventós 2008). Pel que fa a la modalitat escrita, els infants bilingües català-castellà amb TDL mostren dificultats de lectura, especialment en la descodificació al començament de la seva escolarització i en la seva comprensió al final (Aguilar-Mediavilla, Buil-Legaz, Pérez-Castelló, Rigo-Carratalà i Adrover-Roig 2014; BuilLegaz, Aguilar-Mediavilla i Rodríguez-Ferreiro 2015, 2016).

A més, com ja hem indicat abans, els infants amb TDL també mostren dificultats amb habilitats de processament generals. Els estudis existents fets amb nens i nenes bilingües de castellà i català amb TDL han mostrat dificultats persistents en tasques relacionades amb la manipulació de segments de paraules (consciència fonològica) i en el manteniment d'unitats verbals actives en memòria fonològica (repetició de paraules o repetició de 
frases), mentre que altres habilitats, com ara l'accés a les representacions fonològiques subjacents (denominació ràpida o fluïdesa verbal), es veuen afectades de manera primerenca encara que milloren durant el desenvolupament (Buil-Legaz, AguilarMediavilla i Adrover-Roig 2016). Altres estudis en nens i nenes bilingües amb TDL que tenien el castellà com una de les seves llengües també han mostrat dèficits en la memòria fonològica (Girbau i Schwartz 2008) i en el processament d'informació auditiva i visual (Pons, Andreu, Sanz-Torrent, Buil-Legaz i Lewkowicz 2013).

\section{DETECCIÓ PRECOÇ, FACTORS DE RISC I SIGNES D’ALERTA}

Si tenim en compte la definició de TDL, que posa en relleu que el problema lingüístic ha de ser persistent, no podrem fer un diagnòstic abans dels 506 anys d'edat, quan se suposa que s'han superat les adquisicions bàsiques per al desenvolupament del llenguatge (McKean, Wraith, Eadie, Cook, Mensah i Reilly 2017). No obstant això, abans d'aquesta edat, podem detectar algunes senyals que ens poden indicar o fer sospitar que ens trobem davant d'una possible dificultat amb el llenguatge, que podria desembocar en un desenvolupament atípic i per tant en un trastorn del llenguatge oral.

En tot cas, sempre hem d'evitar, costi el que costi, el temut «ja parlarà», és a dir, l'estratègia d'esperar a veure què passa (Capone Singleton 2018, Lowry 2018). Cal derivar l'infant a una avaluació, fer-ne una intervenció preventiva i un seguiment tan aviat com se sospiti d'un possible retard del llenguatge. De fet, un de cada cinc infants que comencen a parlar tard mantindrà una dificultat greu de llenguatge a l'edat de 5 anys, i el 40\% presentarà alguna altra dificultat associada (Capone Singleton 2018; Miniscalco, Fernell, Thompson, Sandberg, Kadesjö i Gillberg 2018). A més, la resta d'infants que sembla que milloren el llenguatge no vol dir que s'igualin als seus parells d'edat en aquesta habilitat. De fet, els estudis longitudinals han mostrat que els parlants tardans que milloren el llenguatge cap als 6 anys poden tornar a presentar dificultats quan inicien la lectura (una modalitat diferent però basada en el llenguatge oral; Scarborough i Dobrich 1990). A més a més, també mostren unes competències lingüístiques menors, tant orals com escrites, quan arriben a l'adolescència (Rescorla 2002, 2009; Dale, McMillan, Hayiou-Thomas i Plomin 2014; Hawa i Spanoudis 2014), tot i que és cert que tenen millors competències lingüístiques que aquells que varen mantenir la dificultat de llenguatge als 6 anys (és a dir, que els infants que han estat diagnosticats de TDL; Stothard, Snowling Bishop, Chipchase i Kaplan 1998). A aquest efecte se l'ha anomenat recuperació il-lusòria (Scarborough i Dobrich 1990) i fa referència a un desenvolupament inicialment retardat del llenguatge, fins que es va apropant a la corba dels seus parells caps als 5-6 anys. Però que torna a separar-se'n quan els aprenentatges lingüístics es tornen més complexos o impliquen una nova modalitat (escrita). El retard en l'inici del llenguatge també impacta en les funcions executives, la regulació emocional, la socialització, els resultats acadèmics i posa en risc els parlants tardans a una dificultat a llarg termini, com pot ser la inserció laboral (Hawa i Spanoudis 2014; Capone Singleton 2018).

Per tant, la detecció precoç és fonamental per poder intervenir com més aviat millor i, en la mesura del possible, pal-liar les conseqüències de la dificultat del llenguatge, i que aquestes no es cronifiquin. La premissa de la intervenció precoç es basa en el fet que, en els primers anys de vida, el nen té més plasticitat cerebral quan encara no s'ha consolidat l'adquisició del llenguatge. No hem de pensar que, més enllà d'aquesta edat, la intervenció no sigui possible, però sí que cal saber que serà menys beneficiosa. La intervenció precoç pot impulsar una millora del llenguatge a llarg termini, i evitar l'impacte de les dificultats del llenguatge en altres àmbits que es fonamenten en els èxits lingüístics previs (recordem el neuroconstructivisme; Capone Singleton 2018). Si aconseguim això, el fet de treballar 
durant un període especialment sensible al desenvolupament lingüístic, ens pot permetre solucionar possibles desfasaments cronològics en determinades adquisicions. A més a més, el fet que el nen només presenti un inici tardà del llenguatge no vol dir que no necessiti una intervenció precoç, ni que no se’n pugui beneficiar. Aquesta intervenció l'ajudarà a aconseguir les fites del desenvolupament més fàcilment d'acord amb la seva edat $\mathrm{i}$ a pal-liar altres trastorns comòrbids.

En l'àmbit més funcional, una detecció precoç, fins i tot anterior a l'elaboració del diagnòstic, dota el nen d'eines per cobrir les seves necessitats actuals i millorar el seu dia a dia. Finalment, tenint en compte la dimensió més emocional de les dificultats de llenguatge, una detecció precoç permet que les famílies disminueixin el seu nivell d'ansietat davant la situació d'incertesa que solen viure durant el procés de diagnòstic. Els estudis previs han mostrat que la intervenció en infants parlants tardans (2-4 anys) sense encara un diagnòstic és efectiva i millora les seves habilitats lingüístiques (Cable i Domsch 2011). Els models basats en la interacció social i en els models d'intervenció a partir dels pares, semblen ser els més adequats, ja que es duen a terme en un context natural de l'infant, equilibren els estils interactius entre pares i fills i mostren un augment del vocabulari expressiu quan es compara amb altres tractaments (Capone Singleton 2018, Lowry 2018, Roberts i Kaiser 2015).

Però per poder fer aquesta detecció precoç ens podem ajudar dels factors de risc i els senyals d'alerta dels trastorns del llenguatge. En primer lloc, s'han detectat diversos factors que estan associats amb un risc més elevat de presentar dificultats persistents en l'adquisició del llenguatge, tot i que no s'ha trobat cap predictor únic fiable (Nelson, Nygren, Walker i Panoscha 2006; Berkman, Wallace, Watson, Coyne-Beasley, Cullen, Wood i Lohr 2011). Cal tenir en compte que la revisió de la literatura posa en evidència la dificultat de trobar una llista completament consistent sobre factors de risc específics que, abans dels 5 anys, puguin guiar en la identificació prèvia d'aquests trastorns. No obstant això, es poden destacar els factors de risc següents:

- Sexe masculí. Alguns estudis han mostrat un factor de risc més alt de dificultats lingüístiques a llarg termini en els infants de sexe masculí (Zambrana, Pons, Eadie i Ystrom 2014).

- Història familiar de dificultats lingüístiques, lectores o acadèmiques. Sembla que darrere del trastorn del desenvolupament del llenguatge hi ha un factor genètic amb un factor d'heretabilitat d'entre el 50\% i el $75 \%$ que, a més, és compartit amb altres dificultats d'aprenentatge, com la dislèxia (Bishop 2006). Els estudis longitudinals han mostrat també que els infants amb inici tardà del llenguatge i amb antecedents familiars tenen més risc de presentar un retard persistent (Reilly et al. 2010; Zambrana et al. 2014).

- Prematuritat. El risc de tenir dificultats del llenguatge en prematurs és més alt i augmenta a mesura que disminueix l'edat gestacional, afectant tant la producció com la percepció lingüística (Bosch 2011; McKean, Reilly, Bavin, Bretherton, Cini, Conway, Cook, Eadie, Prior, Wake i Mensah 2017; BoschGalcerán et al. 2018).

- Estatus socioeconòmic baix i nivell acadèmic baix de la mare. Els estudis han mostrat que els nivells baixos d'educació materna i l'estatus socioeconòmic prediuen posteriors resultats més baixos en el llenguatge dels infants (Reilly et al. 2010).

- Estil d'interacció inadequat entre pares i fill. Molts estudis han mostrat un estil interactiu qualitativament diferent, però quantitativament similar, entre els pares d'infants amb retard de la parla i els pares de nens sense dificultats (Girolametto, Weitzman, Wiigs i Pearce 1999; Hawa i Spanoudis 2014). 
Altres variables que més es repeteixen en les publicacions investigades són el baix pes en néixer, el fet pertànyer a una família molt nombrosa, el baix nivell de lectura a la família i haver estat escolaritzat des de molt petit (Berkman et al. 2011; Nelson et al. 2006). També hi ha altres variables que apareixen en la literatura, especialment en el cas de nens prematurs, però amb bastanta menys freqüència i de manera inconsistent. Variables com el context cultural de la família, l'ordre de naixement que hi ocupa l'individu (haver nascut més tard que els germans), l'edat de la mare en néixer el nen (tant si és molt jove com si és molt gran), pares amb pràctiques educatives pobres, l'alt nivell d'estrès parental, dificultats mentals de la mare, l'alcoholisme fetal o la mala alimentació del nadó (Berkman et al., 2011; Nelson et al., 2006).

Cal destacar que és especialment la combinació d'aquests factors de risc, i no només d'un d'aquests, el que fa l'infant més vulnerable a presentar dificultats del llenguatge persistents (Sylvestre, Desmarais, Meyer, Bairati i Leblond 2018). De fet, sembla que els perfils d'agreujament de les dificultats del llenguatge es relacionen amb la presència d'un nombre més elevat de factors de risc ambientals (ser immigrant, tenir un nivell sociocultural baix, tenir pocs llibres i poc hàbit de lectura a casa), mentre que els infants que tenen poca resposta en la intervenció es relacionen amb la presència de factors biològics (baix pes en néixer, problemes socioemocionals i història familiar de dificultats de llenguatge; McKean, Wraith et al. 2017).

També, hi ha diferents signes d'alerta que ens poden estar indicant, ja des de pràcticament el naixement, que el desenvolupament de l'infant no segueix el ritme esperat per la seva edat. Així, entre els signes d'alerta de les dificultats del llenguatge abans dels 5 anys d'edat, cal destacar:

- Nens massa tranquils.

- Repertori consonant limitat. Alguns estudis han mostrat inventaris consonàntics i menor intel-ligibilitat en els infants que mantenen les dificultats de llenguatge amb el pas del temps (Roberts, Rescorla, Giroux i Stevens 1998; Williams i Elbert 2003).

- Balboteig limitat. Oller, Eilers, Neal, i Schwartz (1999) van trobar que els infants que iniciaven més tard el balboteig canònic (típicament iniciat cap als 6 mesos) tenien un vocabulari més reduït als 18,24 i 30 mesos.

- Aparició tardana de les primeres combinacions de paraules, més enllà dels 24 mesos (Rudolph i Leonard 2016).

- Retard en les habilitats de comprensió. Els parlants tardans amb retards en la producció i la comprensió tenen un risc més elevat de patir trastorns lingüístics persistents que els nens que només presenten dificultats expressives (Zambrana et al. 2014).

- Ús limitat del joc simbòlic. Els nens amb dificultats de llenguatge presenten un ús més limitat del joc simbòlic, amb menys episodis, menys esquemes i amb un desenvolupament menor de les seqüències de joc, a l'edat de 24 mesos (Rescorla i Goossens 1992).

- Manca o ús reduït de gestos de representació. Thal, Tobias, i Morrison (1991) varen mostrar que els parlants tardans que mantenien el seu retard de llenguatge un any després de detectar aquest retard, utilitzaven menys gestos a l'edat de 2 i 3 anys, en comparació amb parlants tardans que l'havien solucionat. En canvi, a edats superiors ( 5 i 10 anys) l'ús de gestos icònics per reemplaçar paraules, sobretot substituir substantius, és superior en els infants amb TDL que en els seus parells d'edat (Blake, Myszczyszyn, Jokel i Bebiroglu 2008).

- Desenvolupament molt lent del llenguatge expressiu. En molts estudis longitudinals l'inici tardà de les primeres vocalitzacions, així com el propi 
desenvolupament alentit del llenguatge, s'han mostrat com a predictors de les dificultats posteriors del llenguatge. Les investigacions mostren que entre el $50 \%$ i el $70 \%$ dels infants amb dificultats amb el llenguatge que ja comencen a parlar als 24 mesos tendeixen a igualar el seu perfil als seus parells d'edat, mentre que el $82 \%$ dels que als 30 mesos encara no parlaven tenien problemes lingüístics persistents als 6 anys (Hawa i Spanoudis 2014). Sembla també que un desenvolupament gramatical pobre als 3 anys (per exemple, mesurat amb la longitud mitjana de l'enunciat) és un factor de pronòstic del nivells de llenguatge posterior (Sylvestre et al. 2018).

- Manca d'imitació verbal. La limitada imitació de frases i de no paraules s'ha proposat com un marcador del trastorn del desenvolupament del llenguatge en moltes llengües, independent a variables socials i lingüístiques (Conti-Ramsden et al. 2001). Les principals dificultats es troben en els verbs, els clítics i els continguts molt llargs (Girbau i Schwartz 2008, Smolík i Vávrů 2014).

- Vocabulari expressiu que consisteix principalment en substantius amb pocs o nuls verbs. Alguns estudis han mostrat que els nens amb trastorns del llenguatge tenen un repertori molt petit o no utilitzen pràcticament verbs (Jones i Conti-Ramsden 1997).

- Habilitats socials pobres. Els parlants tardans mantenen dificultats socials inclús havent solucionat els problemes expressius (Paul, Spangle, Looney, i Dahm 1991).

En canvi, alguns factors que tradicionalment s'havien proposat de risc per les dificultats del llenguatge han trobat evidència negativa d'associació amb les dificultats del llenguatge. Per exemple, en el cas de la història personal d'infeccions d'oïda o otitis. Els estudis mostren evidència negativa entre la presència d'otitis els primers anys de vida i els resultats lingüístics posteriors (Roberts, Rosenfeld i Zeisel 2004; Schwartz et al. 1998). 


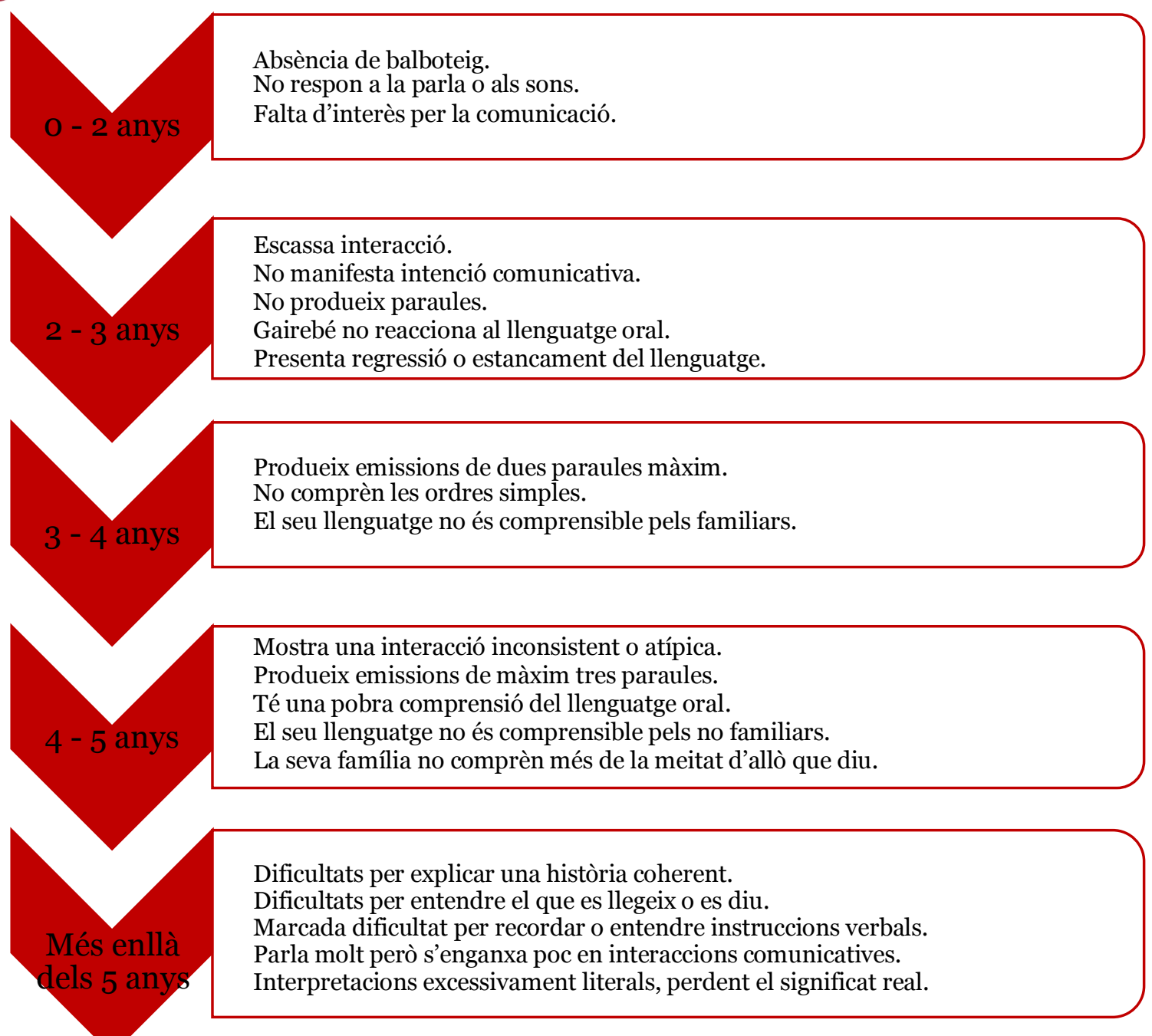

Font: Bishop et al. 2016

Aquests signes d'alerta es poden organitzar per edats, seguint les fites evolutives pròpies de cada etapa del desenvolupament, observant si l'infant fa allò que s'espera dels nens de la seva edat en cadascuna de les etapes d'adquisició lingüística. La Figura 1 mostra els signes d'alerta de dificultats del llenguatge segons el projecte CATALISE (Bishop et al. 2016).

La primera impressió diagnòstica, o la sospita de dificultats derivada de l'observació dels signes d'alerta en infants tan petits, ha de ser revisada amb freqüència, mantenint el seguiment del nen o nena per comprovar-ne l'evolució després de la intervenció. Així, un diagnòstic dinàmic -en el qual s'avalua, s'intervé i es torna a avaluar per observar-ne l'evolució- és fonamental en tot moment, però especialment a aquestes edats primerenques, ja que permet ajustar el diagnòstic en funció de l'evolució i és el millor predictor de l'evolució del nen. Típicament, els infants amb inici tardà del llenguatge, però sense trastorn, normalitzen ràpidament les seves habilitats lingüístiques després d'un breu període d'intervenció, mentre que en els casos de trastorns del llenguatge, tot i que la intervenció millora el llenguatge del nen, aquest continua presentant dificultats (Lidz i Peña 2009; Petersen, Chanthongthip, Ukrainetz, Spencer i Steeve 2017).

En resum, la literatura suporta un model de factors de risc del TDL en què com més quantitat de factors de risc presents, especialment si estan presents des de molt aviat, més 
risc de presentar un retard persistent del llenguatge. Per tant, la presència de més factors de risc indica una necessitat més gran d'intervenció clínica (Bishop 2014; Hawa i Spanoudis 2014). En paraules de Bishop (Bishop 2006):

«El llenguatge sol ser sorprenentment robust davant circumstàncies de desenvolupament adverses. Això suggereix que hi pot haver diverses rutes per a l'adquisició efectiva del llenguatge, i si una ruta està bloquejada, generalment es pot trobar una altra. No obstant això, si es bloquegen dos o més itineraris, l'aprenentatge del llenguatge es veurà compromès».

\section{REFERÈNCIES BIBLIOGRÀFIQUES}

Adrover-Roig, Daniel; SÁnchez-AZAnza, Víctor; López-Penadés, Raül (2018). «Trastornos asociados a un déficit en las funciones ejecutivas y dificultades del lenguaje». Aguilar-Mediavilla, Eva; Igualada, Alfonso (ed.). Dificultades del habla, el lenguaje y la comunicación en los trastornos del neuro-desarrollo. Barcelona: UOC.

Aguilar-Mediavilla, Eva; Buil-Legaz, lucía; Pérez-Castelló, Josep A; RigoCARRATALÀ, EDUARD; ADROVER-ROIG, DANIEL (2014). «Early preschool processing abilities predict subsequent reading outcomes in bilingual Spanish-Catalan children with Specific Language Impairment (SLI)». Journal of Communication Disorders, 50, 19-35.

Aguilar-Mediavilla, Eva; Sanz-Torrent, Mònica; Serra-Raventós, Miquel (2002). «A comparative study of the phonology of pre-school children with Specific Language Impairment (SLI), Language Delay (LD) and Normal Acquisition». Clinical Linguistics \& Phonetics, 16(8), 573-596.

- - (2007). «Influence of phonology on morpho-syntax in Romance languages in children with Specific Language Impairment (SLI)». International Journal of Language and Communication Disorders, 43(3), 325-347.

Aguilar-Mediavilla, Eva; Serra-Raventós, Miquel (2006). «Phonological profile of Spanish-Catalan children with specific language impairment at age 4: are there any changes over time?». Folia Phoniatrica et Logopaedica, 58, 400-414.

Alvarado, Helena; Pérez-Castelló, Josep A (2018). «Definición y clasificación de los trastornos específicos de aprendizaje». Aguilar-Mediavilla, Eva; Igualada, Alfonso (ed.). Dificultades del habla, el lenguaje y la comunicación en los trastornos del neurodesarrollo. Barcelona: UOC.

ARChibald, Lisa MD; Gathercole, Susan E (2006). «Prevalence of SLI in Language Resource Units». Journal of Research in Special Educational Needs, 6(1), 3-10.

ARchibald, Lisa MD; Oram Cardy, Janis; Joanisse, Marc F; ANSARi, Daniel (2013). «Language, reading, and math learning profiles in an epidemiological sample of school age children». PLOS ONE, 8(10), 1-13.

Berkman, Nancy D; Wallace, Ina; Watson, linda; Coyne-Beasley, Tamera; Cullen, KatiE; WoOD, ChARLES; LOHR, KATHLEEN N (2011). «Screening for Speech and Language Delays and Disorders in Children Age 5 Years or Younger: A Systematic Review for the U.S. Preventive Services Task Force». Evidence Synthesis No. 12O. AHRQ Publication No. 13o5197-EF-1. Rockville, MD: Agency for Healthcare Research and Quality.

BISHOP, DOROTHY VM (1992). «The underlying nature of specific language impairment.». Journal of Child Psychology and Psychiatry, 33(1), 3-66.

- (2006). "What Causes Specific Language Impairment in Children?». Current directions in psychological science, 15(5), 217-221.

-- (2014). «Ten questions about terminology for children with unexplained language problems». International Journal of Language and Communication Disorders, 49(4), 381-415.

Del trastorn específic del llenguatge (TEL) al trastorn del desenvolupament del llenguatge 80 (TDL): un canvi de concepció sobre els trastorns del llenguatge 
- - (2017). "Why is it so hard to reach agreement on terminology? The case of developmental language disorder (DLD)». International Journal of Language \& Communication Disorders, 1-10.

Bishop, Dorothy VM; Snowling, Margaret J; Thompson, Paul A; Greenhalgh, Trisha; CATALISE-2 CONSORTIUM (2017). "Phase 2 of CATALISE: A multinational and multidisciplinary Delphi consensus study of problems with language development: Terminology». The Journal of Child Psychology and Psychiatry, 58(10), 1068-1080.

- (2016). «CATALISE : a multinational and multidisciplinary Delphi consensus study. 1. Identifying language impairments in children». PLOS ONE, 11(7), 1-26.

Blake, JoAnna; Myszczyszyn, Debbie; JoKel, ARIELA; Bebiroglu, NedA (2008). «Gestures accompanying speech in specifically language-impaired children and their timing with speech». First Language, 28(2), 237-253.

Bosch-Galcerán, Laura; Agut QuiJano, Thais; Busquets Ferrer, Lourdes (2018). «Prematuridad y dificultades del lenguaje». Aguilar-MEdiavilla, Eva; Igualada, ALFONSo (ed.). Dificultades del habla, el lenguaje y la comunicación en los trastornos del neuro-desarrollo. Barcelona: UOC.

BOSCH, LAURA (2011). «Precursors to language in preterm infants». BRADDICK, OLIVER; ATKINSON, JANETTE; INNOCENTI, GIORGIO M. (ed.). Gene Expression to Neurobiology and Behavior: Human Brain Development and Developmental Disorders. Elsevier Science, 239-257.

Buil-Legaz, lucía; Aguilar-Mediavilla, Eva; Adrover-Roig, Daniel (2016). «Longitudinal trajectories of the representation and access to phonological information in bilingual children with specific language impairment». International Journal of SpeechLanguage Pathology, 18(5), 473-482.

Buil-Legaz, lucía; Aguilar-Mediavilla, Eva; Rodríguez-Ferreiro, Javier (2015). «Reading skills in young adolescents with a history of Specific Language Impairment: The role of early semantic capacity». Journal of Communication Disorders, 58, 14-20.

- - (2016). «Oral morphosyntactic competence as a predictor of reading comprehension in children with specific language impairment». International Journal of Language \& Communication Disorders, 51(4), 473-477.

CABle, Amory LaW; Domsch, Celeste (2011). «Systematic review of the literature on the treatment of children with late language emergence». International Journal of Language and Communication Disorders, 46(2), 138-154.

CAPone Singleton, Nina (2018). «Late Talkers: Why the Wait-and-See Approach Is Outdated». Pediatric Clinics of North America, 65(1), 13-29.

CAtTs, Hugh W; Fey, Marc E; Tomblin, J Bruce; Zhang, XuYang (2002). «A longitudinal investigation of reading outcomes in children with language impairments». Journal of Speech, Language, and Hearing Research, 45(6), 1142.

Conti-Ramsden, Gina; Botting, Nicola; Faragher, Brian (2001). «Psycholinguistic markers for Specific Language Impairment (SLI)». Journal of Child Psychology and Psychiatry, 42(6), 741-748.

D’SouZA, Dean; FilipPI, Roberto (2017). «Progressive modularization: Reframing our understanding of typical and atypical language development». First Language, 37(5), 518529 .

Dale, Philip S; McMillan, Andrew J; Hayiou-Thomas, Marianna E; Plomin, Robert (2014). "Illusory Recovery: Are Recovered Children With Early Language Delay at Continuing Elevated Risk?». American Journal of Speech-Language Pathology, 23(3), 437-447. 
FAZIO, BARBARA B (1996). «Mathematical Abilities of Children With Specific Language Impairment». Journal of Speech Language and Hearing Research, 39(4), 839.

GIRBAU, DOLORS; SCHWARTZ, RICHARD G (2008). «Phonological working memory in Spanish-English bilingual children with and without specific language impairment». Journal of communication disorders, 41(2), 124-145.

Girolametto, Luigi; Weitzman, Elaine; Wiigs, Megan; Pearce, Patsy Steig (1999). «The relationship between maternal language measures and language development in toddlers with expressive vocabulary delays». American Journal of Speech-Language Pathology, 8(4), 364-374.

GOUlandris, NATA K; SNOWLing, MARgaret J; WALKER, IAN (2000). «Is dyslexia a form of specific language impairment? A comparison of dyslexic and language impaired children as adolescents». Annals of Dyslexia, 50(1), 103-120.

HAWA, VERA V.; SPANOUdIS, GEORGE (2014). «Toddlers with delayed expressive language: An overview of the characteristics, risk factors and language outcomes». Research in Developmental Disabilities, 35(2), 400-407.

Hegde, Mahabalagiri N. (1996). A coursebook on language disorders in children. Singular Pub. Group.

HeNRY, LuCy A; MesSer, DAVID J; NASH, Gilly (2012). «Executive functioning in children with specific language impairment». Journal of Child Psychology and Psychiatry and Allied Disciplines, 53(1), 37-45.

Hill, Elisabeth L (1998). «A dyspraxic deficit in specific language impairment and developmental coordination disorder? Evidence from hand and arm movements». Developmental Medicine and Child Neurology, 40(6), 388-395.

HiLl, ElisabeTh L; Bishop, DVM; NimMo-SMiTH, I (1998). « Representational gestures in developmental co-ordination disorder and specific language impairment: Error-types and the reliability of ratings». Human Movement Science, 17(4-5), 655-678.

JOHNSTON, JUDITH R (1999). «Cognitive deficits in specific language impairment: Decision in spite of uncertainty». Journal of Speech-Language Pathology and Audiology, 23(4), 165-172.

Jones, Melanie; Conti-Ramsden, Gina (1997). «A comparison of verb use in children with SLI and their younger siblings». First Language, 17(51), 165-184.

KARMILOFF-SMith, ANNETTE (1998). "Development itself is the key to understanding developmental disorders». Trends in Cognitive Science, 2(10), 389-398.

LEONARD, LAURENCE B (1998). Children with specific language impairment. Massachusetts: MIT Press (MA).

LidZ, Carol S; PeÑa, Elizabeth D (2009). «Response to Intervention and Dynamic Assessment: Do We Just Appear to Be Speaking the Same Language?». Seminars in Speech and Language, 213(02), 121-134.

LINDSAY, GEOFF; STRAND, STEVE (2016). «Children with Language Impairment: Prevalence, Associated Difficulties, and Ethnic Disproportionality in an English Population». Frontiers in Education, 1:2.

LOWRY, LAUREN (2018). «Don't wait-and-see, research suggests». Hanen Early Language Program. <https://www.hanen.org/SiteAssets/Articles---Printer-Friendly/Research-inyour-Daily-Work/Dont-wait-and-see-research-suggests-PF.aspx $>$ [Consulta: 27 maig 2019].

Maggio, Verónica; Grañana, Nora E; Richaudeau, Alba; Torres, Silvio; Giannotti, ADRIÁN; SUBURO, ANGELA M (2014). «Behavior problems in children with specific language 
impairment». Journal of Child Neurology, 29(2), 194-202.

MARTON, KLARA (2008). «Visuo-spatial processing and executive functions in children with specific language impairment». International Journal of Language \& Communication Disorders, 43(2), 181-200.

McKean, Cristina; Reilly, Sheena; Bavin, Edith L; Bretherton, Lesley; Cini, Eileen; CONWAY, LAURA; ... MENSAH, FIONA (2017). «Language outcomes at 7 years: Early predictors and co-occurring difficulties». Pediatrics, 139(3), e20161684.

McKean, Cristina; Wraith, Darren; Eadie, Patricia; Cook, Fallon; Mensah, Fiona; REILLY, SHEENA (2017). «Subgroups in language trajectories from 4 to 11 years: the nature and predictors of stable, improving and decreasing language trajectory groups». Journal of Child Psychology and Psychiatry, 10, 1081-1091.

Miniscalco, Carmela; Fernell, Elisabeth; Thompson, Lucy; Sandberg, Eva; Kadesjö, BJÖRN; GILLBERG, CHRISTOPHER (2018). «Development problems were common five years after positive screening for language disorders and, or, autism at 2.5 years of age». Acta Paediatrica, International Journal of Paediatrics, 107(10), 1739-1749.

MONTGOMERY, JAMES W (2003). «Working memory and comprehension in children with specific language impairment: what we know so far». Journal of Communication Disorders, 36(3), 221-231.

Morton, J BRUCE (2005). Understanding Developmental Disorders. Oxford: Blackwell Publishing.

MuELLER, KATHYRn L; TOMBLin, J BRUCE (2012). «Exmaining the comorbidity of language disorders and ADHD». Biological Bulletin, 221(1), 18-34.

Nelson, HD; Nygren, Peggy; Walker, Miranda; Panoscha, Rita (2006). «Screening for Speech and Language Delay in Preschool Children: Systematic Evidence Review for the US Preventive Services Task Force». Pediatrics, 117(2), e298-e319.

Oller, D Kimbrough; Eilers, Rebecca E; Neal, A Rebecca; Schwartz, Heidi K (1999). «Precursors to speech in infancy: The prediction of speech and language disorders». Journal of Communication Disorders, 32(4), 223-245.

Paul, Rhea; Spangle Looney, Shwan; Dahm, S Pamela (1991). «Communication and Socialization Skills at Ages 2 and 3 in «Late-Talking» Young Children». Journal of Speech and Hearing Research, 34(August), 858-865.

Petersen, Douglas B; Chanthongthip, Helen; Ukrainetz, Teresa A; Spencer, Trina D; STEEVE, ROGER W (2017). «Dynamic assessment of narratives: Efficient, accurate identification of language impairment in bilingual students». Journal of Speech, Language and Hearing Research, 6o(April), 983-998.

Pons, Ferran; Andreu, Llorenç; SAnz-Torrent, Mònica; Buil-Legaz, Lucía; LEWKOWICZ, DAVID J (2013). «Perception of audio-visual speech synchrony in Spanishspeaking children with and without specific language impairment». Journal of Child Language, 40(3), 687-700.

Reilly, Sheena; Wake, Melissa; Ukoumunne, Obioha C; Bavin, Edith; Prior, Margot; Cini, EILEEN; ... BRETHERTON, LESLEY (2010). «Predicting language outcomes at 4 years of age: findings from Early Language in Victoria Study.». Pediatrics, 126(6), e1530-7.

ResCORLA, LeSLIE (2002). «Language and Reading Outcomes to Age 9 in Late-Talking Toddlers». Journal of Speech, Language, and Hearing Research, 45(2), 360-371.

- - (2009). «Age 17 Language and Reading Outcomes in Late-Talking Toddlers: Support for a Dimensional Perspective on Language Delay». Journal of Speech Language and Hearing Research, 52(1), 16. 
RESCORLA, Leslie; Goossens, MariJKe (1992). «Symbolic Play Development in Toddlers With Expressive Specific Language Impairment (SLI-E)». Journal of Speech Language and Hearing Research, 35(6), 1290.

Roberts, JoAnne E; Rosenfeld, Richard M; Zeisel, SusAn A (2004). «Otitis media and speech and language: a meta-analysis of prospective studies». Pediatrics, 113(3 Pt 1), e23848.

Roberts, JULIE; RESCORLA, LeSLIE; GIROUX, JeNNIFER; STEVENS, LiSA (1998). «Phonological skills of children with specific expressive language impairment (SLI-E): Outcome at age 3 ». Journal of Speech, Language and Hearing Research, 41, 374-384.

RoberTs, MEgan Y; KAISER, ANn P (2015). «Early intervention for toddlers with language delays: A randomized controlled trial». Pediatrics, 135(4), 686-693.

Rocha-Muniz, Caroline Nunes; Befi-Lopes, DÉbora Maria; Schochat, Eliane (2015). «Mismatch negativity in children with specific language impairment and auditory processing disorder». Brazilian Journal of Otorhinolaryngology, 81(4), 408-415.

RudolPh, Johanna M; LEONARD, LAURENCE B (2016). «Early language milestones and specific language impairment». Journal of Early Intervention, 38(1), 41-58.

SANZ-Torrent, Mònica; BAdia, Iris; Serra-Raventós, Miquel (2008). «Contributions from bilingual language impairment in Catalan and Spanish to the understanding of typical and pathological language acquisition». En Carmen Pérez Vidal Maria Juan-Garau \& Aurora Bel (Ed.), A Portrait of the Young in the New Multilingual Spain (p. 135-158). Clevedon: Multilingual matters.

Sanz-Torrent, Mònica; Serrat Sellabona, Elisabet; Andreu Barrachina, Llorenç; SERRA-RAVENTÓs, MiQUeL (2008). «Verb morphology in Catalan and Spanish in children with SLI: a developmental study.». Clinical Linguistics and Phonetics, 22(6), 459-474.

SCARborough, Hollis S; Dobrich, WANDA (1990). «Development of Children with Early Language Delay». Journal of Speech Language and Hearing Research, 33(1), 70.

SCHWARTZ, Richard G; Mody, MARIA; PETINOU, KAKIA (1998). «Phonological acquisition in children with OME: Speech perception and speech production». ROBERTS, JOANNE E.; Wallace, Ina F; Henderson, Frederick W. (ed.). Otitis Media in Young Children: Medical, Developmental, and Educaational Considerations. Baltimore, MD: Paul H. Brookes Publishing.

SERRA-RAVENTÓs, Miquel (2002). «Trastornos del lenguaje: preguntas pendientes en investigación e intervención». Revista de logopedia, foniatría y audiología, 22(2), 63-76.

Serra-Raventós, Miquel; Aguilar-Mediavilla, Eva; Sanz-Torrent, Mònica (2002). «Evolución del perfil productivo en el trastorno del lenguaje». Revista de Logopedia, Foniatría y Audiología, 22(2), 77-89.

SMOLÍK, FILIP; VÁvrŮ, PeTra (2014). "Sentence imitation as a marker of SLI in Czech: Disproportionate impairment of verbs and clitics». Journal of Speech, Language, and Hearing Research, 57(3), 837-849.

St Clair, Michelle C; Pickles, Andrew; Durkin, Kevin; Conti-Ramsden, Gina (2011). «A longitudinal study of behavioral, emotional and social difficulties in individuals with a history of specific language impairment (SLI)». Journal of Communication Disorders, 44(2), 186-199.

STARK, RE; TAlLal, PaUla (1981). «Selection of children with specific language deficits». Journal of Speech and Hearing Disorders, 46(2), 114-122.

Stothard, Susan E; Snowling, Margaret J; Bishop, Dorothy VM; Chipchase, Barry B; KAPLAN, ChIPCHASE A (1998). "Language-impaired preschoolers: A follow-up into adolescence». Journal of Speech, Language, and Hearing Research, 41(2), 407. 
Sylvestre, Audette; Desmarais, Chantal; Meyer, François; Bairati, Isabelle; Leblond, JEAN (2018). «Prediction of the outcome of children who had a language delay at age 2 when they are aged 4: Still a challenge». International Journal of Speech-Language Pathology, 20, 731-744.

Thal, Donna; Tobias, Stacy; Morrison, Deborah (1991). «Language and Gesture in Late Talkers». Journal of Speech Language and Hearing Research, 34(3), 604.

Williams, A LynN; ElBerT, MARY (2003). «A prospective longitudinal study of phonological development in late talkers». Language, Speech, and Hearing Services in Schools, 34(2), 138-153.

Woods, Patricia LPL; Rieger, Ingrid; Wocadlo, Crista; Gordon, Adrienne (2014). «Predicting the outcome of specific language impairment at five years of age through early developmental assessment in preterm infants». Early Human Development, 90(10), 613619.

Zambrana, Imac Maria; Pons, Francisco; Eadie, Patricia; Ystrom, Eivind (2014). «Trajectories of language delay from age 3 to 5: Persistence, recovery and late onset». International Journal of Language and Communication Disorders, 49(3), 304-316.

ZELAZNIK, HowARD N; GOFFMAN, LiSA (2010). «Generalized motor abilities and timing behavior in children with specific language impairment». Journal of Speech Language and Hearing Research, 53(2), 383. 